\title{
Unraveling how electronic and spin structures control macroscopic properties of manganite ultra-thin films
}

\author{
Xinmao Yin ${ }^{1,2}$, Muhammad Aziz Majidi ${ }^{1,2,3}$, Xiao Chi ${ }^{1,2}$, Peng Ren ${ }^{4}$, Lu You ${ }^{4}$, Natalia Palina ${ }^{2}$, Xiaojiang $\mathrm{Yu}^{2}$, \\ Caozheng Diao ${ }^{2}$, Daniel Schmidt ${ }^{2}$, Baomin Wang ${ }^{5}$, Ping Yang ${ }^{2}$, Mark BH Breese ${ }^{1,2}$, Junling Wang ${ }^{4}$ \\ and Andrivo Rusydi ${ }^{1,2}$
}

Perovskite manganites exhibit fascinating transport and magnetic properties, essential for fundamental research and applications. With the development of thin film technologies, more exotic properties have been observed in doped-manganites over a wide range of temperature. Unraveling the interplay of spin, charge and orbital degrees of freedom that drives exotic, macroscopic properties is therefore crucial for the understanding of strongly correlated electron systems. Here, using a combination of transport, spectroscopic ellipsometry, X-ray absorption spectroscopy and X-ray magnetic circular dichroism, we observe two concomitant electronic and magnetic phases (insulating paramagnetic phase for $T>195 \mathrm{~K}$ and insulating cantedferromagnetic for $T<140 \mathrm{~K}$ ) with an intermediate metal-like state in ultra-thin $\mathrm{La}_{0.7} \mathrm{Sr}_{0.3} \mathrm{MnO}_{3}$ (LSMO) film on DyScO ${ }_{3}$ substrate. Surprisingly, the $02 p-M n 3 d$ hybridization strength reduces with decreasing temperature, driving the system more insulating and ferromagnetic. The Jahn-Teller effect weakens markedly within the intermediate temperature range, making the system more metal-like. We also apply this comprehensive method to a $\mathrm{LSMO}$ film on $\mathrm{SrTiO}_{3}$ substrate for comparison. Our study reveals that the interplay of the $02 p-M n 3 d$ hybridization and the dynamic Jahn-Teller splitting controls the macroscopic transport and magnetic properties in ultra-thin manganites.

NPG Asia Materials (2015) 7, e196; doi:10.1038/am.2015.65; published online 3 July 2015

\section{INTRODUCTION}

Perovskite manganites exhibit fascinating transport and magnetic properties, essential for fundamental research and applications. ${ }^{1-7}$ In particular, with the development of thin film nanoscale technologies, more exotic properties have been observed in doped-manganite thin films over a wide range of temperature. ${ }^{8-21}$ However, the origin of many fundamental phenomena remains unclear. For instance, it has been recently shown that doped-manganite ultra-thin films have anomalous transport anisotropic properties, and such complex phenomena could not be explained with a strain effect alone. ${ }^{20}$ This raises an important, fundamental question of the role of spin, charge and orbital to macroscopic properties in doped-manganite ultra-thin films and strongly correlated electron systems.

Here, we design a new, unique approach to unravel the detailed evolution of electronic and spin structures and their relationship to macroscopic transport and magnetic properties versus temperature. A doped-manganite, $\mathrm{La}_{0.7} \mathrm{Sr}_{0.3} \mathrm{MnO}_{3}$ (LSMO) ultra-thin film on a $\mathrm{DyScO}_{3}$ (DSO) substrate (LSMO/DSO) is chosen as a model system. Using a combination of temperature-dependent spectroscopic ellipsometry, X-ray absorption spectroscopy (XAS), and X-ray magnetic circular dichroism (XMCD) both at $\mathrm{O} K$-edge and $\mathrm{Mn} L_{3,2}$-edges as well as transport measurements, we show for the first time how the interplay of hybridization, orbital occupancy and spin controls the macroscopic properties. Two concomitant electronic and magnetic phases (insulating paramagnetic phase for $T>195 \mathrm{~K}$ and insulating canted-ferromagnetic for $T<140 \mathrm{~K}$ ) with an intermediate metal-like state are observed. Surprisingly, the $\mathrm{O} 2 \mathrm{p}-\mathrm{Mn} 3 d$ hybridization strength reduces with decreasing temperature, driving the system to become more insulating-like and ferromagnetic. The Jahn-Teller effect weakens markedly within the intermediate temperature range, turning the system into a metal-like state. From our measurements, the temperature-dependent $\mathrm{O} 2 p-\mathrm{Mn} 3 d$ hybridization strength, the energy splitting of $e_{\mathrm{g}}$ by the Jahn-Teller effect and its band width are extracted. From these we propose the temperature-dependent density of states of the system.

To further demonstrate how our comprehensive methodology works, we also present a study on LSMO ultra-thin films on $\mathrm{SrTiO}_{3}$ substrates (LSMO/STO) for comparison. We observe that LSMO/STO behaves differently from that on LSMO/DSO, despite both being under tensile strain, of similar thickness and the same chemical

${ }^{1}$ NUSSNI-NanoCore, Department of Physics, National University of Singapore (NUS), Singapore, Singapore; ${ }^{2}$ Singapore Synchrotron Light Source (SSLS), National University of Singapore (NUS), Singapore, Singapore; ${ }^{3}$ Departemen Fisika, FMIPA, Universitas Indonesia, Depok, Indonesia; ${ }^{4}$ School of Materials Science and Engineering, Nanyang Technological University (NTU), Singapore, Singapore and ${ }^{5}$ Key Laboratory of Magnetic Materials and Devices, Ningbo Institute of Material Technology and Engineering, Chinese Academy of Sciences, Zhejiang, P. R. China

Correspondence: Professor J Wang. School of Materials Science and Engineering, Nanyang Technological University (NTU), Singapore 639798, Singapore.

E-mail: jlwang@ntu.edu.sg

or Professor A Rusydi, Department of Physics, National University of Singapore, 2 Science Drive 3, Singapore 117542, Singapore.

E-mail: phyandri@nus.edu.sg

Received 17 January 2015; revised 20 March 2015; accepted 20 April 2015 

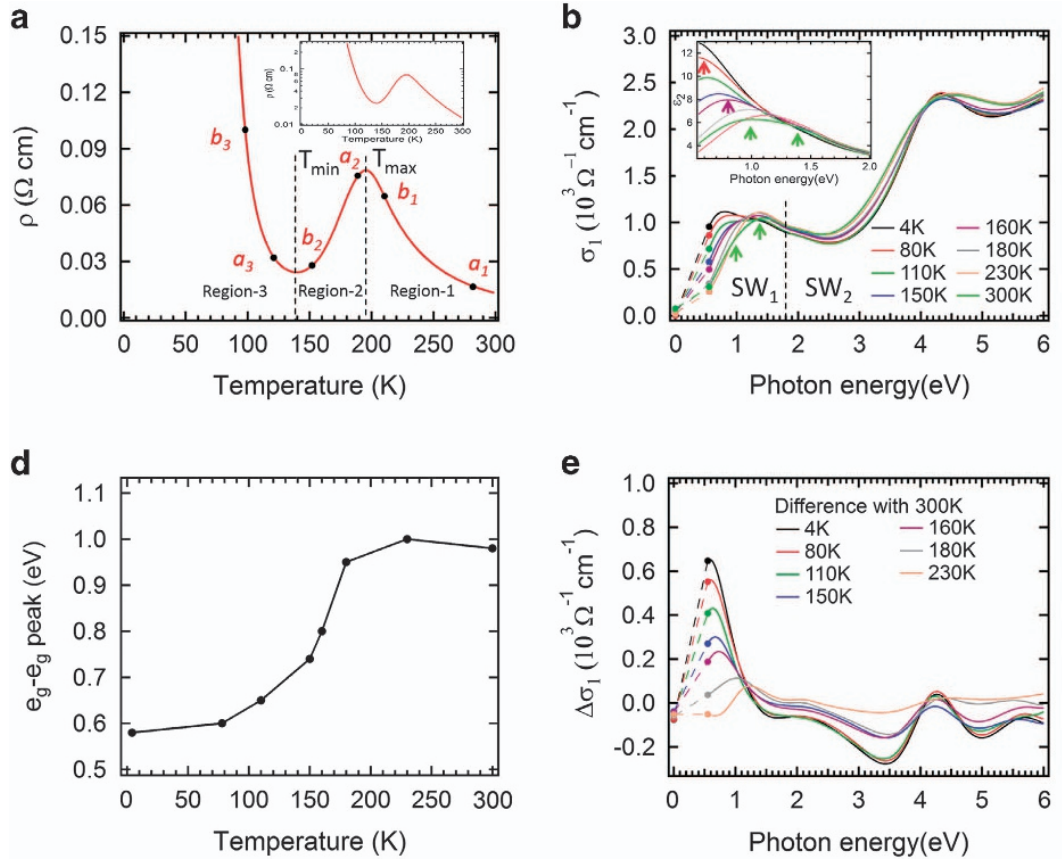

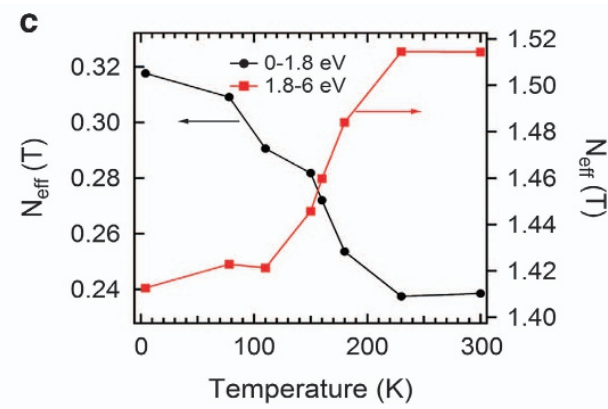

f

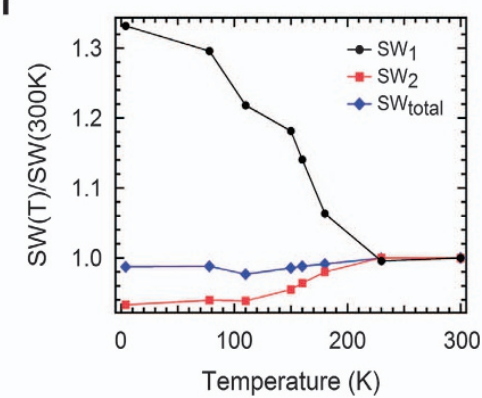

Figure 1 Transport and spectroscopic ellipsometry on ultra-thin $\mathrm{La}_{0.7} \mathrm{Sr}_{0.3} \mathrm{MnO}_{3}$ film. (a) Resistivity $\left(\rho\right.$ ) versus temperature curve for the $\mathrm{La}_{0.7} \mathrm{Sr}_{0.3} \mathrm{MnO}_{3}$ film on $\mathrm{DyScO}_{3}$ substrate (LSMO/DSO) along [001]-orthorhombic direction for DyScO 3 . Two transition temperatures are observed, $T_{\min }(\sim 140 \mathrm{~K})$ and $T_{\max }$ ( 195 K). Region-1: $T>T_{\max }$; Region-2 (or intermediate): $T_{\min }<T<T_{\max }$ Region-3: $T<T_{\min }$. The inset is the resistivity vs temperature on log scale. (b) Optical conductivity $\left(\sigma_{1}(\omega)\right)$ from 0 to $6 \mathrm{eV}$ as a function of temperature for ultra-thin LSMO/DSO film. The $\sigma_{1}$ at energy between $0 \mathrm{eV}$ (estimated from the conductivity in (a) and $0.55 \mathrm{eV}$ ) is estimated using a linear interpolation. The two light-green color arrows point to the energy position of $\mathrm{Mn}-e_{\mathrm{g}} \rightarrow \mathrm{Mn}-\mathrm{e}_{\mathrm{g}}$ transition $(\sim 1 \mathrm{eV})$ and of charge transfer $02 p \rightarrow \mathrm{Mn}-e_{\mathrm{g}}$ transition $(\sim 1.4 \mathrm{eV})$ at $300 \mathrm{~K}$. The inset is the imaginary part of the dielectric function $\varepsilon_{2}(\omega)$ from 0.55 to $2 \mathrm{eV}$ as a function of temperature. The different color arrows point to the energy position of the $e_{\mathrm{g}}-e_{\mathrm{g}}$ transition at $80 \mathrm{~K}$ (red), $160 \mathrm{~K}$ (purple) and $300 \mathrm{~K}$ (light green). (c) The number of effective charge ( $N_{\text {eff }}$ ) as a function of temperature for two different regions: 0-1.8 and 1.8-6 eV. (d) Energy position of the Jahn-Teller peak ( $e_{\mathrm{g}}-e_{\mathrm{g}}$ transition) versus temperature obtained from $\mathbf{b}$ and $\mathbf{e}$ ) Change of optical conductivity $\Delta \sigma_{1}(T)$ defined as $\sigma_{1}(T)-\sigma_{1}(300 \mathrm{~K})$ for LSMO/DSO. (f) Integrated spectral weight $\frac{S W(T)}{S W(300 K)}$ defined as $\frac{\int_{\omega_{1}}^{\omega_{2}} \sigma_{1}(\omega, T) d \omega}{\int_{\omega_{1}}^{\omega_{1}} \sigma_{1}(\omega, T=300 K) d \omega}$ in different spectral regions: $S W_{1}(0-1.8 \mathrm{eV}), S W_{2}(1.8-6 \mathrm{eV})$, and total energy range $\mathrm{SW}_{\text {total }}(0-6 \mathrm{eV}) . T$ is temperature (Kelvin).

doping/stoichiometry. Here, although the $\mathrm{O} 2 p-\mathrm{Mn} 3 d$ hybridization strength increases with decreasing temperature, our similar scenario explains well the observed macroscopic properties.

\section{MATERIALS AND METHODS}

\section{Samples growth}

High-quality epitaxial LSMO thin films are grown by pulsed laser deposition on atomically smooth [110]-orthorhombic oriented DSO and [001]-cubic oriented STO single-crystal substrate. ${ }^{20}$ The laser pulse $(248 \mathrm{~nm})$ energy density was $\sim 2 \mathrm{~J} \mathrm{~cm}^{-1}$ and the repetition rate was $3 \mathrm{~Hz}$. The growth was carried out under 200 mTorr oxygen partial pressure at $800^{\circ} \mathrm{C}$ and the growth rate was $\sim 0.8 \mathrm{~nm} \mathrm{~min}^{-1}$.

High-resolution X-ray diffractometry and structure characterization The crystallographic structure of LSMO films on DSO and STO substrates is characterized by high-resolution X-ray diffractometry in the X-ray Demonstration and Development beamline at the Singapore Synchrotron Light Source. High-resolution X-ray diffractometry studies reveal a perfectly coherent interface between the film and substrate (see Supplementary Figures 1, 2 and 3). The measured lattice constants of LSMO film (on DSO) are monoclinic $a=0.3938(3) \mathrm{nm}, b=0.3955(3) \mathrm{nm}, c=0.3831(1) \mathrm{nm}, \beta=92.79(8)^{\circ}$. The thickness is estimated to be $12.6 \mathrm{~nm}$. The larger lattice constant of the DSO substrate leads to tensile strain in the film, reducing the cla ratio of LSMO to 0.968. Meanwhile, for the LSMO/STO sample, we obtained a value of $c / a$ of $0.995(<1)$. This system is also under tensile strain (see Supplementary information for details).

\section{Electrical measurements}

In-plane transport properties of the films are investigated using a lowtemperature probe station system at temperatures ranging from 80 to $300 \mathrm{~K}$. Pt electrodes with dimensions of $0.4 \mathrm{~mm} \times 0.8 \mathrm{~mm}$ are deposited on the film (see Supplementary Figure 4a) through a shadow mask.

Spectroscopic ellipsometry measurements and optical conductivity We use a spectroscopic ellipsometer with a photon energy of $0.55-6 \mathrm{eV}$ to measure the ellipsometry parameters $\Psi$ (the ratio between the amplitude of p- and s-polarized reflected light) and $\Delta$ (the phase difference between of p- and s-polarized reflected light) with a $70^{\circ}$ incident angle and a $45^{\circ}$ polarizer angle (see Supplementary Figure $4 \mathrm{~b}$ ). The dielectric function and optical conductivity $\left(\sigma_{1}\right)$ have been extracted from the parameters $\Psi$ and $\Delta$ by utilizing an air/LSMO/DSO multilayer model (see supplementary information for details $)^{22}$. The $\sigma_{1}$ at $0 \mathrm{eV}$ can be estimated from dc conductivity in Figure 1a. The $\sigma_{1}$ at between 0 and $0.55 \mathrm{eV}$ are estimated using linear interpolation. ${ }^{23}$

\section{X-ray absorption spectroscopy}

The $\mathrm{O} K$-edge absorption spectra in the energy range $520-580 \mathrm{eV}$ and $\mathrm{Mn}$ $L$-edge absorption spectra in the energy range $630-680 \mathrm{eV}$ were obtained using linearly polarized XAS from the Surface, Interface and Nanostructure Science beamline at Singapore Synchrotron Light Source, using a total electron yield detection method. The incidence angle $(\theta)$ of X-rays refers to the normal of the sample surface, which was varied by rotating the polar angle of the sample. The Ell[001]-orthorhombic direction spectra were measured in a normal-incident alignment $\left(\theta=0^{\circ}\right)$ (see Supplementary Figure 4a). The temperature was varied between 80 and $300 \mathrm{~K}$. The spectra were normalized to the integrated intensity 
between 565 and $580 \mathrm{eV}$ for $\mathrm{O} 1 s$ spectra and between 670 and $680 \mathrm{eV}$ for $\mathrm{Mn} 2 p$ spectra after subtracting an energy-independent background.

\section{$\mathrm{X}$-ray linear dichroism measurements}

For $\mathrm{Mn}$, the $3 d_{x^{2}-y^{2}}$ and $3 d_{3 z^{2}-r^{2}}$ orbitals have lobes pointing parallel and perpendicular to the $a b$ plane, respectively. X-ray linear dichroism measurements can probe the occupancy of the $3 d_{x^{2}-y^{2}}$ and $3 d_{3 z^{2}-r^{2}}$ orbitals using linearly polarized light aligned to the out-of-plane (grazing incidence, $\mathrm{E} \sim \| c)$ and in-plane (normal incidence, Ella) directions (Supplementary Figure 4a). The $\mathrm{X}$-ray linear dichroism measurements result shows a near temperature independence at 80 and $300 \mathrm{~K}$ (Supplementary Figure 8). Smaller absorption for in-plane polarization spectra suggests more out-of-plane empty states in the $3 d e_{\mathrm{g}}$ band (a higher occupancy of in-plane orbitals). The spectral difference $(I($ Ella $)-I(E \| l c)$, linear dichroism) implies a preferential occupancy of the $3 d_{x^{2}-y^{2}}$ orbital at both 80 and $300 \mathrm{~K}$. The result is in good agreement with the theoretically calculated result for LSMO film under tensile strain. ${ }^{18,24}$

\section{$\mathrm{X}$-ray magnetic circular dichroism measurements}

The XMCD signal is the difference between absorption spectra obtained with a $+1 \mathrm{~T}$ and a $-1 \mathrm{~T}$ magnetic field, which was set parallel to the X-ray beam direction using plus helicities of circular polarized light. The grazing-incident spectra were measured at $\theta=60^{\circ}$ (see Supplementary Figure 4a), which is estimated as the magic angle. ${ }^{25,26}$ The degree of circular polarization $\left(P_{\mathrm{c}}\right)$ was calculated to be $88 \%$.

\section{RESULTS}

\section{Transport properties}

The temperature-dependent resistivity of the LSMO film along the in-plane [001]-orthorhombic direction (for the DSO substrate) reveals unusual behavior with two transition-temperatures, $T_{\min }(\sim 140 \mathrm{~K})$ and $T_{\max }(\sim 195 \mathrm{~K})$ (Figure 1a). Insulating behavior is observed both above $T_{\max }$ and below $T_{\min }$, whereas a metal-like state appears in between (at the intermediate temperature region). To reveal the microscopic mechanism behind these transitions, we use a combination of techniques including temperature-dependent spectroscopic ellipsometry, XAS and XMCD to probe the detailed electronic and spin structures directly.

\section{Electronic properties}

Let us first discuss results from spectroscopic ellipsometry measurements (see Figures 1b-f). The spectroscopic ellipsometer reveals optical conductivity $\left(\sigma_{1}(\omega)\right)$ (see Methods), which directly connects to the electronic density of states (as discussed later). As shown in previous studies, by measuring $\sigma_{1}(\omega)$ in a broad energy range, one can find the origin of charges that contribute to transport measurements. ${ }^{22,23}$ Figure $1 \mathrm{~b}$ displays a combination of $\sigma_{1}(\omega)$ spectra (from 0.55 to $6 \mathrm{eV}$ ) and transport measurements $(0 \mathrm{eV})$, showing strong temperature dependence of $\sigma_{1}(\omega)$ in a broad energy range. An enhancement of spectral weight of $\sigma_{1}(\omega)$ below $1.8 \mathrm{eV}$ on cooling is coming from spectral weight above $1.8 \mathrm{eV}$. This implies the importance of electronic structure of higher energy bands determining low-energy transport properties. This will be clear in the following detailed analysis. For simplicity, $\sigma_{1}(\omega)$ is divided into two regions: below $1.8 \mathrm{eV}$ (low-energy region, $\mathrm{SW}_{1}$ for spectral weight) and above $1.8 \mathrm{eV}$ (high-energy region, $\mathrm{SW}_{2}$ for spectral weight). The number of effective charges $\left(N_{\text {eff }}\right)$ can be quantified as follows:

$$
N_{\mathrm{eff}}=\frac{2 m_{e} V}{\pi e^{2}} \int_{\omega_{1}}^{\omega_{2}} \sigma\left(\omega^{\prime}\right) d \omega^{\prime} .
$$

In our measured spectral energy range, we find that the total number of charges is nearly constant (within $\sim 1.2 \%$ ) as a function of temperature (Figure 1c). As charge conservation has to be fulfilled, this is important to validate our next analysis. ${ }^{22,23}$ As shown in Figure 1c, the enhancement of $N_{\text {eff }}(T)$ in $\mathrm{SW}_{1}$ as temperature decreases is closely accompanied by the reduction of $N_{\text {eff }}(T)$ in $\mathrm{SW}_{2}$. This directly tells us that the low-energy transport property is determined by electronic bands at higher energy bands.

We inspect now in more detail the low-energy region. Two peaks are observed at $\sim 1.4$ and $\sim 1 \mathrm{eV}$, with different temperaturedependent behavior (see also inset of Figure $1 \mathrm{~b}$ ). The $\sim 1.4 \mathrm{eV}$ peak is almost temperature-independent. This peak has been ascribed to the $\mathrm{O} 2 p \rightarrow$ Mn- $e_{\mathrm{g}}$ excitations. ${ }^{27,28}$ The $\sim 1 \mathrm{eV}$ peak, on the other hand, increases its intensity markedly as the temperature decreases. Interestingly, the position of this peak shows a red-shift between $T_{\max }$ and $T_{\min }$, and remains almost unchanged outside this region (Figures $1 \mathrm{~b}$ and e). According to the above observations and comparing with previous studies, this $\sim 1 \mathrm{eV}$ peak is assigned to the $\mathrm{Mn}-e_{\mathrm{g}} \rightarrow \mathrm{Mn}-e_{\mathrm{g}}\left(\right.$ or $e_{\mathrm{g}}-e_{\mathrm{g}}$ ) transitions with a parallel spin, which is directly related to the Jahn-Teller effect of the Mn3d states. ${ }^{28-31}$ The red-shift of the $\sim 1 \mathrm{eV}$ peak shows that the dynamic Jahn-Teller splitting energy is significantly reduced as the temperature decreases and this happens in the intermediate temperature region (see Figure 1d). Although the origin of the sudden decrease in the Jahn-Teller splitting energy is not clear at the moment, as discussed later, we show that the interplay between the dynamic Jahn-Teller splitting and hybridizations is responsible for the macroscopic property in the intermediate state. Together with the transport data, it is clear that the increase of $\mathrm{SW}_{1}$ does not develop into a Drude response, instead it reveals the dynamics of the $e_{\mathrm{g}}-e_{\mathrm{g}}$ transitions, yielding to insulating behavior at lower temperature. The features in the high-energy region (above $1.8 \mathrm{eV}$ ) in Figure $1 \mathrm{~b}$ arise from transitions between $\mathrm{O} 2 p$ and $\mathrm{Mn} 3 d$ as well as between lower and upper Hund's rule split bands. ${ }^{29,32}$

Owing to the Kramers-Kronig transformation, the pre-eminence of the spectroscopic ellipsometry approach allows one to measure the charge transfer accurately using the optical f-sum rule. ${ }^{22,23}$ As the temperature decreases, an increase of $\mathrm{SW}_{1}$ by as much as $\sim 33 \%$ below $T_{\max }$ is accompanied by a decrease of $\mathrm{SW}_{2}$ spanning an energy range up to $6 \mathrm{eV}$ (Figure 1f). It is found that the total spectral weight (or $N_{\text {eff }}$ ) is nearly conserved throughout the whole-temperature range (Figure 1f (or Figure 1c)). The occurrence of spectral-weight transfer in such a broad energy range as a function of temperature is an important signature of strong electronic correlations and hybridization strength that drives the system from one phase to another. ${ }^{23,33,34}$ Later, it is shown that the spectral weight transfer is due to marked changes of hybridization strength measured using temperaturedependent XAS.

\section{Hybridizations and orbital occupancy}

We next identify details of the hybridization strength, in particular $\mathrm{O} 2 p-\mathrm{Mn} 3 d$ hybridization, and orbital occupancies as functions of temperature and later explain their intimate connection with macroscopic properties. These can be probed directly using element-specific XAS as a function of temperature. ${ }^{33}$ Our main observation is the strong temperature-dependent XAS at O K-edge for Ell[001]orthorhombic direction (Figure $2 \mathrm{a})$. The change of XAS, $\Delta \mu(T)$, is defined as $\mu(T)-\mu(300 \mathrm{~K})$, where $\mu(T)$ is the XAS at temperature $T$. Because the $\mathrm{O} K$-edge XAS reveals transitions from $\mathrm{O} 1 s$ to unoccupied states related to $\mathrm{O} 2 p$ states hybridized with metal states, it is directly related to the corresponding hybridization strengths. In Figure $2 \mathrm{a}$, the $\mathrm{O} K$-edge spectra is divided into three regions: (1) a pre-edge region attributed to $\mathrm{O} 2 p-\mathrm{Mn} 3 d$ hybridization (527-533.5 eV); (2) a broad structure associated with the hybridization of $\mathrm{O} 2 p-\mathrm{La} 5 d$ and $\mathrm{O} 2 p-\mathrm{Sr} 4 d$ 

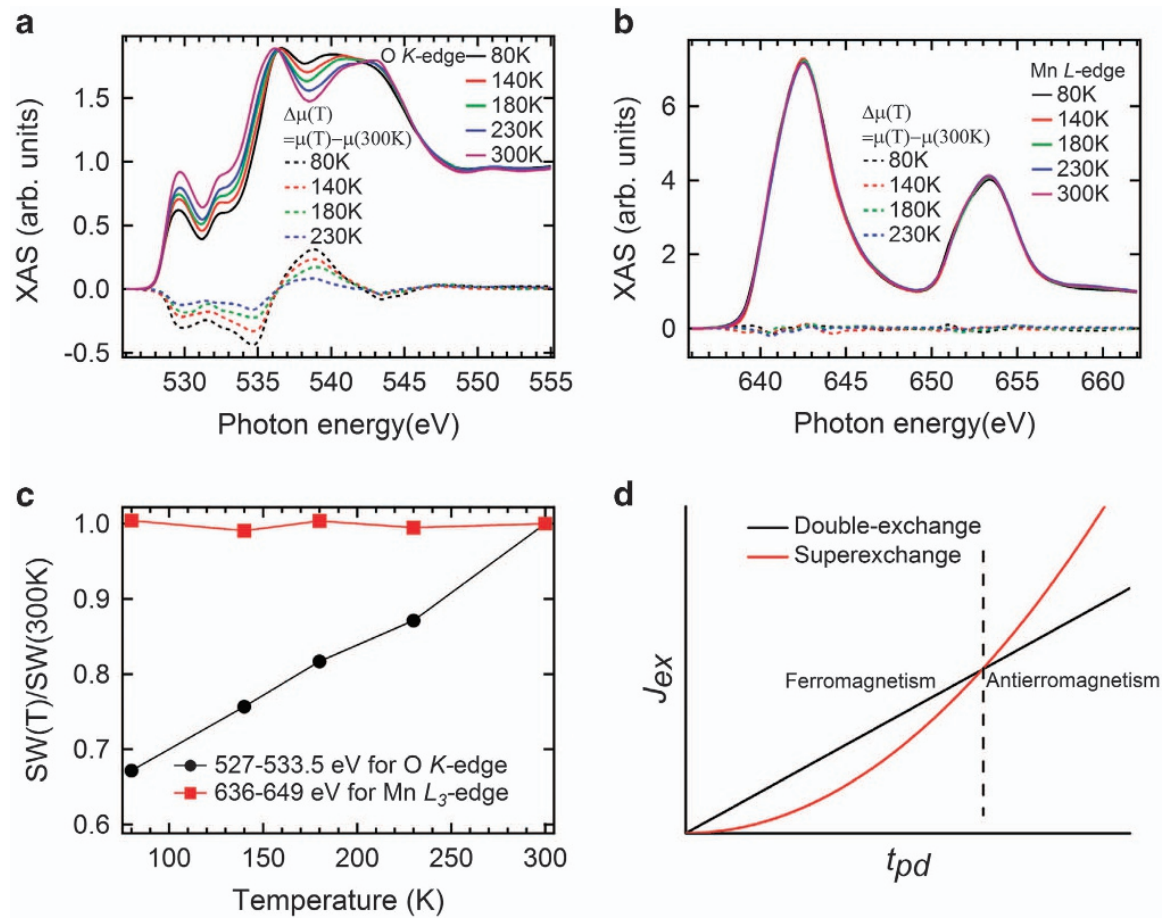

Figure $2 \mathrm{X}$-ray absorption spectra and magnetic structure on ultra-thin $\mathrm{La}_{0.7} \mathrm{Sr}_{0.3} \mathrm{MnO}_{3}$ film. (a) $\mathrm{O}$ K-edge and (b) $\mathrm{Mn} \mathrm{L}_{3,2}$-edge X-ray absorption spectra of the $\mathrm{La}_{0.7} \mathrm{Sr}_{0.3} \mathrm{MnO}_{3}$ film on $\mathrm{DyScO}_{3}$ substrate as a function of temperature for Ell[001]-orthorhombic direction. The change of absorption spectra $\Delta \mu(T)$ defined as $\mu(T)-\mu(300 \mathrm{~K})$ is presented below each spectrum. $\mu(T)$ is the absorption spectrum at temperature $T$. (c) Integrated spectral weight $\frac{S W(T)}{S W(300 K)}$ defined as $\frac{\int_{\omega_{1}}^{\omega_{2}} \mu(\omega, T) d \omega}{\int_{\omega_{1}}^{\omega_{1}} \mu(\omega, T=300 K) d \omega}$ in the energy range of $527-533.5 \mathrm{eV}$ for $0 \mathrm{~K}$-edge spectra and in the energy range of 636-649 eV for Mn $\mathrm{L}_{3}$-edge spectra. (d) The exchange spin coupling $\left(J_{\text {ex }}\right)$ versus hybridization (hopping) between the $02 p$ and Mn3d orbitals $\left(t_{\mathrm{pd}}\right)$. For double exchange, $J_{\mathrm{DE}} \propto t_{\mathrm{pd}}$. For super-exchange, $J_{\mathrm{SE}} \propto\left(t_{\mathrm{pd}}\right)^{2} / U$, where $U$ is a Hubbard energy for $\mathrm{Mn}$.

states (533.5-538.5 eV); and (3) a broad feature due to $\mathrm{O} 2 p$ hybridized with higher energy metal states, for example, $\mathrm{O} 2 p-\mathrm{Mn} 4 s, p$ and $\mathrm{O} 2 p$ La6s,p (538.5-548.5 eV). The observed strong reduction of spectral weight in the pre-edge region as temperature decreases corresponds to a decrease of $\mathrm{O} 2 p-\mathrm{Mn} 3 d$ hybridization strength. ${ }^{35}$

In contrast, the XAS at Mn- $L 3,2$ edges (Figure 2b) are nearly temperature-independent. At the edges, they reflect $\mathrm{Mn} 2 p_{j} \rightarrow \mathrm{Mn} 3 d$ transitions, thus they are directly related to the occupancy of Mn3d orbitals. ${ }^{35}$ The spectra show two broad separated features at $\sim 642$ and $\sim 653 \mathrm{eV}$ owing to strong spin-orbit coupling that splits the $\mathrm{Mn} 2 p_{j}$ core level with $j=3 / 2$ and $1 / 2 .{ }^{35}$ This implies that the change of Mn3d occupancy $\Delta \mu(T)$ is negligible as a function of temperature. This is further supported with temperature-dependent X-ray linear dichroism measurements (Supplementary Figure 9). The relative strength of $\mathrm{O} 2 p-\mathrm{Mn} 3 d$ hybridization (from the pre-edge region in the $\mathrm{O} \mathrm{K}$-edge XAS in Figure 2a) and the occupancy of Mn3d (from the Mn $L_{3,2}$-edge XAS in Figure 2b) as functions of temperature are summarized in Figure $2 \mathrm{c}$. We argue that the spectral weight transfer observed in spectroscopic ellipsometry is due to the change of $\mathrm{O} 2 p-\mathrm{Mn} 3 d$ hybridization strength, in which hybridization together with dynamic Jahn-teller effects control anomalous transport and magnetic properties as discussed later.

\section{Magnetic properties}

The magnetic behavior of LSMO/DSO is probed by element-specific temperature-dependent XMCD at the $\mathrm{Mn} L_{3,2}$-edges and $\mathrm{O} K$-edge (see Methods). Figure 3 shows XMCD at $\mathrm{Mn} L_{3,2}$-edges and $\mathrm{O} \mathrm{K}$-edge at selected temperatures and calculated magnetic moments (spin, orbital and total) (see also supplementary Figure 11 and Supplementary Table 1). At $300 \mathrm{~K}$, there is no observable XMCD signal. The system can be considered to be in a paramagnetic phase. On cooling down to $160 \mathrm{~K}$, where the system behaves as metal-like, the XMCD signal appears. This suggests the appearance of a net magnetic moment. At $80 \mathrm{~K}$, the film displays a further enhanced XMCD signal, only at Mn $L_{3,2}$-edges. As XMCD contains both net spin $\left(m_{\text {spin }}\right)$ and orbital $\left(m_{\text {orb }}\right)$ magnetic moments, one can estimate the total magnetic moment, $m_{\text {total }}=m_{\text {spin }}+m_{\text {orb }}$ as shown in Figure $3 \mathrm{~d}^{36,37}$ Using the angle-dependent XMCD sum rule, ${ }^{25,36,37}$ $m_{\text {total }}$ is found to be $0.202 \mu_{\mathrm{B}}$ at $160 \mathrm{~K}$ and $1.201 \mu_{\mathrm{B}}$ at $80 \mathrm{~K}$ (see supplementary information for details). We find that the total magnetic moment of LSMO/DSO at $80 \mathrm{~K}$ is less than half of that of ferromagnetic bulk LMSO or other LSMO films. ${ }^{38-40}$ Note that such transport and magnetic properties cannot be explained by the strain effect.

\section{DISCUSSION}

By combining spectroscopic ellipsometry and XAS, we uncover the following facts. As the temperature decreases, the $\mathrm{O} 2 p-\mathrm{Mn} 3 d$ hybridization strength $\left(t_{\mathrm{pd}}\right)$ decreases monotonically (as shown by the O K-edge XAS), whereas the occupancy of Mn3d remains almost unchanged (as shown by the Mn $L_{3,2}$-edges XAS). The Jahn-Teller splitting energy of the $e_{\mathrm{g}}$ bands remains relatively unchanged at high and low temperatures, but drops markedly between $T_{\min }$ and $T_{\max }$ (as shown by spectroscopic ellipsometry in Figures $1 \mathrm{~b}$ and $\mathrm{d}$ ).

The anomalous transport behavior (Figure 1a) can now be explained (Figure 4$)$. The $p$ - $d$ hybridization strength $\left(t_{\mathrm{pd}}\right)$ determines 
a
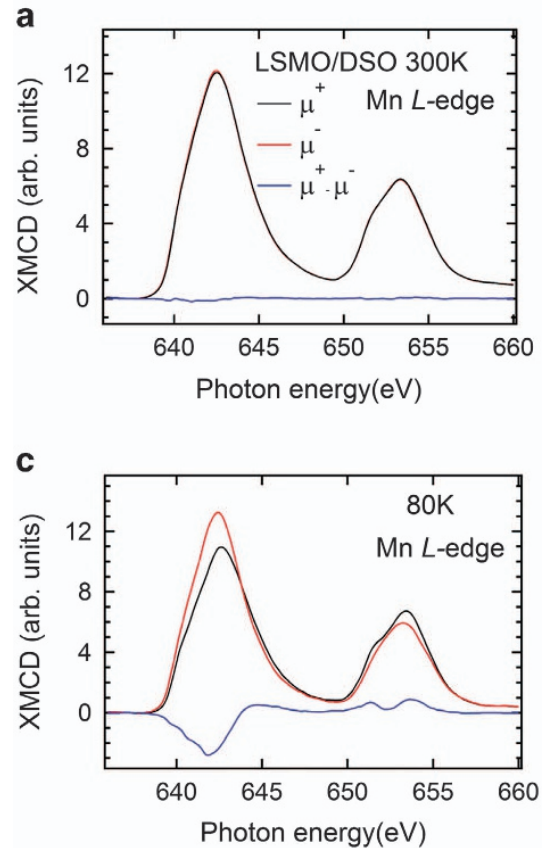

b

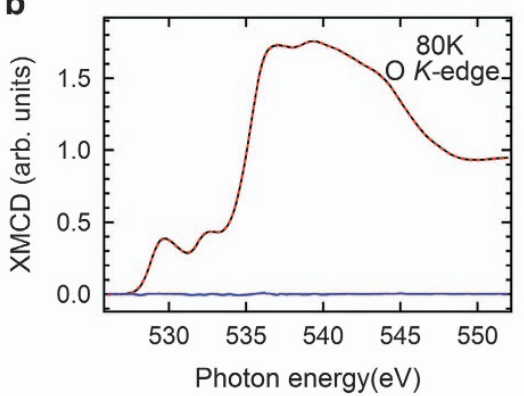

d

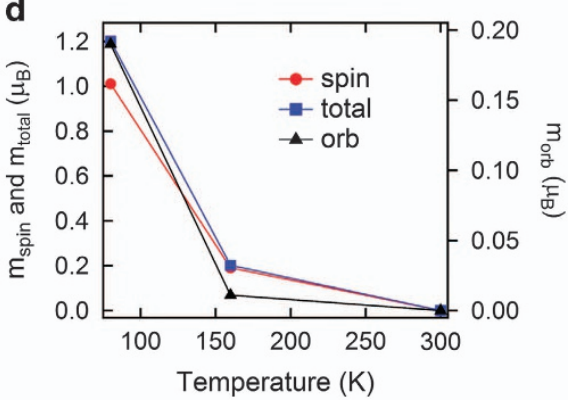

Figure $3 \mathrm{X}$-ray magnetic circular dichroism (XMCD) on ultra-thin $\mathrm{La}_{0.7} \mathrm{Sr}_{0.3} \mathrm{MnO}_{3}$ film. Temperature-dependent XMCD of the $\mathrm{La}_{0.7} \mathrm{Sr}_{0.3} \mathrm{MnO}_{3}$ film on DyScO ${ }_{3}$ substrate at (a, c) Mn $L_{3,2}$-edges and (b) 0 K-edge at selected temperature for grazing incident $\left(\theta=60^{\circ}\right) . \mu^{+}$and $\mu^{-}$are X-ray absorption spectra with two opposite magnetization directions relate to the fixed photon helicity, and the corresponding XMCD signal defined as $\mu^{+}-\mu^{-}$. (d) Magnetic moment of spin $\left(m_{\text {spin }}\right)$, orbital $\left(m_{\text {orb }}\right)$ and total $\left(m_{\text {total }}\right)$ is calculated from XMCD at $M n L_{3,2}$ as a function of temperature.

a
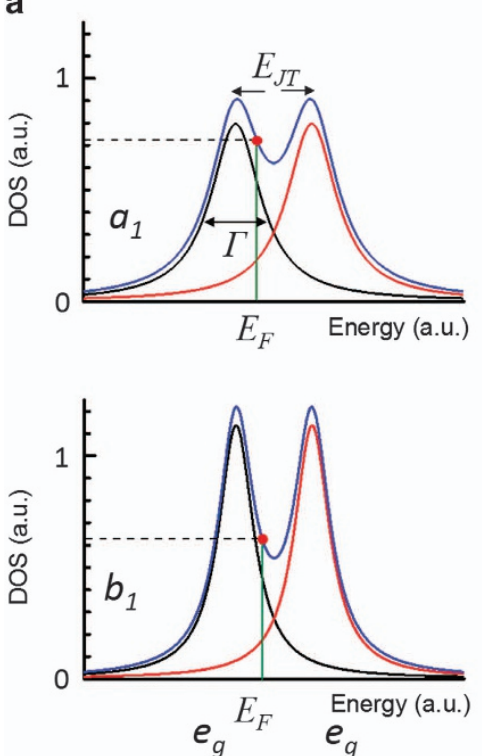

$t_{p d}^{a_{1}}>t_{p d}^{b_{1}}, \quad \Gamma^{a_{1}}>\Gamma^{b_{1}}$

$E_{J T}^{a_{1}} \approx E_{J T}^{b_{1}}$

$\operatorname{DOS}^{a_{1}}\left(E_{F}\right)>\operatorname{DOS}^{b_{1}}\left(E_{F}\right)$

$\rho^{a_{1}}<\rho^{b_{1}}$

Region-1

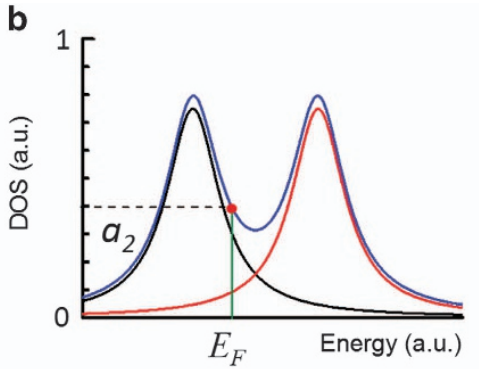

C
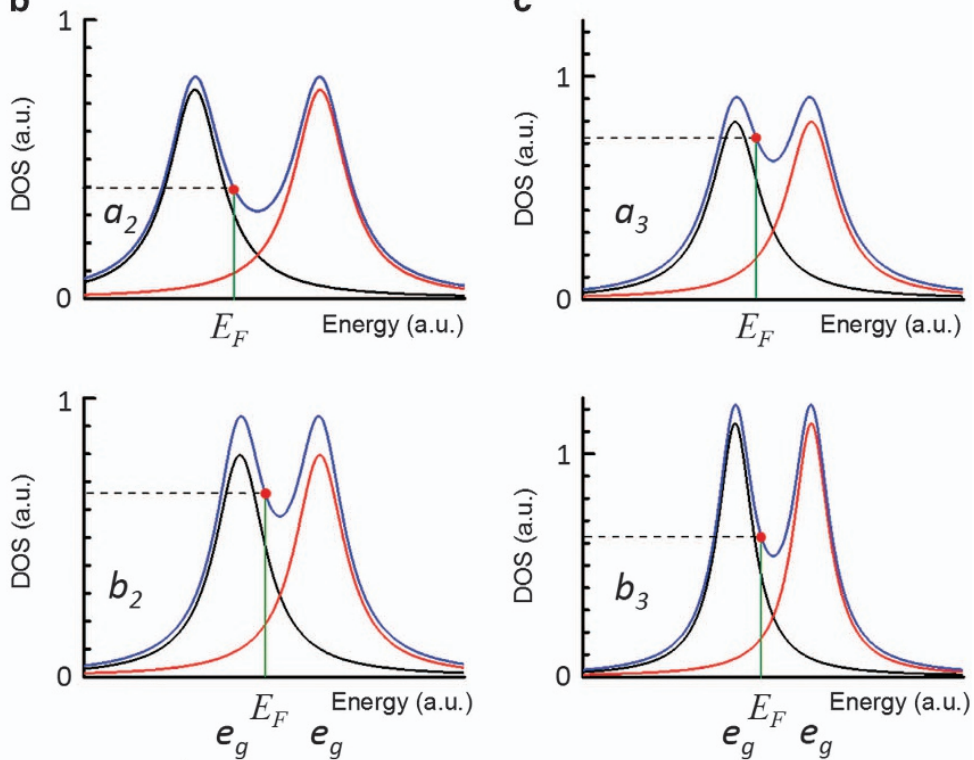

$t_{p d}^{a_{2}}>t_{p d}^{b_{2}}, \quad \Gamma^{a_{2}}>\Gamma^{b_{2}}$

$t_{p d}^{a_{3}}>t_{p d}^{b_{3}}, \quad \Gamma^{a_{3}}>\Gamma^{b_{3}}$

$E_{J T}^{a_{2}}>E_{J T}^{b_{2}}$

$\operatorname{DOS}^{a_{2}}\left(E_{F}\right)<\operatorname{DOS}^{b_{2}}\left(E_{F}\right)$

$\rho^{a_{2}}>\rho^{b_{2}}$

Region-2 
the bandwidths $(\Gamma)$ of the two Jahn-Teller-split $e_{g}$ bands, between which the Fermi level is located. For $T>T_{\max }$ (see Figure $4 \mathrm{a}$ ), as $T$ decreases $t_{\mathrm{pd}}$ decreases causing $\Gamma$ to decrease, which in turn decreases the density of states at the Fermi level $\left(\operatorname{DOS}\left(E_{\mathrm{F}}\right)\right)$. As resistivity $(\rho)$ is inversely proportional to this quantity, thus $\rho$ increases. For $T_{\min }<T<T_{\max }$ (see Figure $4 \mathrm{~b}$ ), while $t_{\mathrm{pd}}$ keeps decreasing, the Jahn-Teller effect weakens more rapidly (as indicated by the abrupt decrease in the Jahn-Teller splitting energy $\left(E_{\mathrm{JT}}\right)$ ), causing the $\operatorname{DOS}\left(E_{\mathrm{F}}\right)$ to increase, thus $\rho$ decreases, transforming the system to metal-like. For $T<T_{\min }$ (see Figure $4 \mathrm{c}$ ), $t_{\mathrm{pd}}$ continues to decrease, whereas $E_{\mathrm{JT}}$ is almost unchanged, causing the $\operatorname{DOS}\left(E_{\mathrm{F}}\right)$ to decrease, thus $\rho$ increases, turning the system back to insulating. Clearly that it is the competition between $\mathrm{O} 2 p-\mathrm{Mn} 3 d$ hybridization and dynamic Jahn-Teller effect that sparks the non-monotonic temperature-dependent transport behavior. Neither one of them alone can explain the observation.

Concomitantly, the change of magnetic behavior as $T$ decreases is also affected by $t_{\mathrm{pd}}$. It is well known that super-exchange $\left(J_{\mathrm{SE}}\right)$ and double-exchange $\left(J_{\mathrm{DE}}\right)$ couplings can be related to the hopping parameter (in our case $t_{\mathrm{pd}}$ ) following $J_{\mathrm{SE}} \propto \frac{t_{\mathrm{pd}}^{2}}{U}$ and $J_{\mathrm{DE}} \propto t_{\mathrm{pd}}$, respectively. This is illustrated by the $J_{\mathrm{ex}}$ vs $t_{\mathrm{pd}}$ sketch in Figure $2 \mathrm{~d}$, demonstrating that antiferromagnetism is favorable for large $t_{\mathrm{pd}}$ (high $T$ ), whereas ferromagnetism is favorable for small $t_{\mathrm{pd}}$ (low $T$ ). Although super-exchange normally favors antiferromagnetism and double-exchange favors ferromagnetism ${ }^{41}$, in their close proximity the competition may lead to a canted-ferromagnetic (or canted-antiferromagnetic) spin arrangement.

As discussed above, our XMCD measurement reveals a small net magnetization for the ultra-thin LSMO film on DSO at low temperature. One might think of a phase separation between ferromagnetic and antiferromagnetic or other non-magnetic phases. ${ }^{42,43}$ We argue that this is not the case here for the following reasons. First, we do not observe a XMCD signal at the $\mathrm{O} K$-edge (see Supplementary Figure 11), suggesting that the local ferromagnetic regions are not present in our samples. As a comparison, for ferromagnetic bulk LSMO, the dichroism at the $\mathrm{O} K$-edge is significant, emphasizing the importance of the double-exchange mechanism. ${ }^{40}$ Second, temperature-dependent resistivity measurements on similar LSMO/DSO ultra-thin film show no hysteresis between cooling and heating at the low-temperature region, ${ }^{20}$ which further supports a single-phase character. Thus, a cantedferromagnetic (or canted-antiferromagnetic) phase is the most plausible interpretation for the low-temperature magnetic phase of LSMO/DSO ultra-thin films.

Combining the above analyses, we can conclude the following. At $T=300 \mathrm{~K}$, the system is paramagnetic owing to the strong thermal fluctuation. Upon cooling, $t_{\mathrm{pd}}$ decreases (Figure 2c) accompanied by the appearance of a net magnetic moment (Figures $3 \mathrm{~b}$ and $\mathrm{c}$ and discussion above), whereas the orbital occupancy of $\mathrm{Mn} 3 d$ remains constant (Figure 2c). This suggests that the spins start to align along a certain direction with some degree of canting owing to the competing mechanisms of super-exchange and double exchange. With further cooling down to $80 \mathrm{~K}$, the net magnetic moment increases because $J_{\mathrm{DE}}$ becomes more dominant over $J_{\mathrm{SE}}$ for lower temperature.

To further support our argument on the necessity of looking at the electronic and spin structures directly using the comprehensive methodology, we also present our study on LSMO ultra-thin films on STO substrates, LSMO/STO (see Supplementary Figure 12). Despite being under tensile strain too, the same chemical composition and very similar film thickness, we find that the strength of $p$ - $d$ hybridization increases as temperature decreases (see Supplementary
Figure 12a and the black dot in Supplementary Figure 12b). This is opposite to that observed in LSMO/DSO (see Figure 2a and black dot in Figure 2c), yet it explains well the observed monotonic decrease of the resistivity and increase of magnetization in LSMO/STO as temperature decreases (see Supplementary information for details).

In conclusion, by combining transport, spectroscopic ellipsometry, XAS and XMCD studies, we have revealed clearly the interplay of hybridizations and the dynamic Jahn-Teller effect that determines the macroscopic electronic transport and magnetic properties of LSMO/DSO ultra-thin films. Furthermore, the methodology presented here leading to our conclusion is valuable for the study of other strongly correlated systems.

\section{CONFLICT OF INTEREST}

The authors declare no conflict of interest.

\section{ACKNOWLEDGEMENTS}

This work is supported by Singapore National Research Foundation under its Competitive Research Funding (NRF-CRP 8-2011-06 and NRF2008NRFCRP002024), MOE-AcRF Tier-2 (MOE2010-T2-2-121) and NUS-YIA. We acknowledge the CSE-NUS computing center for providing facilities for our numerical calculations. The work at Nanyang Technological University is supported by National Research Foundation of Singapore under project NRF-CRP5-2009-04. JW acknowledges support from National Natural Science Foundation of China under the Overseas, Hong Kong \& Macau Scholars Collaborated Research Fund (51428201).

Author contributions: X Yin, XC, NP, XY, DC and AR performed X-ray absorption and XMCD measurements. X Yin, DS and AR performed spectroscopic ellipsometry measurements. JW, PR and LY grew high-quality thin films and performed transport measurements. YP, X Yin and AR performed synchrotron X-ray diffraction and topography measurements. MAM and AR performed theoretical calculations. XYin, MAM, AR analyzed the data. AR wrote the manuscript with the assistance from XYin, MAM, JW and MBHB AR planned and designed a combination of spectroscopic ellipsometry, X-ray absorption and XMCD measurements. AR and JW supervised the project.

1 Jonker, G. H. \& Van Santen, J. H. Ferromagnetic compounds of manganese with perovskite structure. Physica 16, 337-349 (1950).

2 Saitoh, E., Okamoto, S., Takahashi, K. T., Tobe, K., Yamamoto, K., Kimura, T. Ishihara, S., Maekawa, S. \& Tokura, Y. Observation of orbital waves as elementan excitations in a solid. Nature 410, 180-183 (2001).

3 Milward, G. C., Calderon, M. J. \& Littlewood, P. B. Electronically soft phases in manganites. Nature 433, 607-610 (2005).

4 Hueso, L. E., Pruneda, J. M., Ferrari, V., Burnell, G., Valdés-Herrera, J. P. Simons, B. D., Littlewood, P. B., Artacho, E., Fert, A. \& Mathur, N. D. Transformation of spin information into large electrical signals using carbon nanotubes. Nature 445 , 410-413 (2007)

5 Cheong, S. W. \& Mostovoy, M. Multiferroics: a magnetic twist for ferroelectricity. Nat. Mater. 6, 13-20 (2007).

6 Basov, D. N., Averitt, R. D., van der Marel, D., Dressel, M. \& Haule, K. Electrodynamics of correlated electron materials. Rev. Mod. Phys. 83, 471-541 (2011).

7 Beaud, P., Caviezel, A., Mariager, S. O., Rettig, L., Ingold, G., Dornes, C., Huang, S. W. Johnson, J. A., Radovic, M., Huber, T., Kubacka, T., Ferrer, A., Lemke, H. T., Chollet, M., Zhu, D., Glownia, J. M., Sikorski, M., Robert, A., Wadati, H., Nakamura, M., Kawasaki, M., Tokura, Y., Johnson, S. L. \& Staub, U A time-dependent order parameter for ultrafast photoinduced phase transitions. Nat. Mater. 13, 923-927 (2014)

8 Izumi, M., Konishi, Y., Nishihara, T., Hayashi, S., Shinohara, M, Kawasaki, M. \& Tokura, Y. Atomically defined epitaxy and physical properties of strained La0.6Sr0.4MnO3 films. Appl. Phys. Lett. 73, 2497-2499 (1998).

9 Sun, J. Z., Abraham, D. W., Rao, R. A. \& Eom, C. B. Thickness-dependent magnetotransport in ultrathin manganite films. Appl. Phys. Lett. 74 3017-3019 (1999).

10 Bowen, M., Bibes, M., Barthelemy, A., Contour, J.-P., Anane, A., Lemaitre, Y. \& Fert, A Nearly total spin polarization in La2/3Sr1/3MnO3 from tunneling experiments. Appl. Phys. Lett. 82, 233-235 (2003).

11 Orgiani, P., Petrov, A. Yu., Adamo, C., Aruta, C., Baruta, C., De Luca, G. M., Galdi, A. Polichetti, M., Zola, D. \& Maritato, L. In-plane anisotropy in the magnetic and transport properties of manganite ultrathin films. Phys. Rev. B 74, 134419 (2006). 
12 Aruta, C., Ghiringhelli, G., Tebano, A., Boggio, N. G., Brookes, N. B., Medaglia, P. G. \& Balestrino, G. Strain induced x-ray absorption linear dichroism in $\mathrm{LaO} .7 \mathrm{SrO} .3 \mathrm{MnO} 3$ thin films. Phys. Rev. B 73, 235121 (2006).

13 Thiele, C., Dörr, K., Bilani, O., Rödel, J. \& Schultz, L. Influence of strain on the magnetization and magnetoelectric effect in La0.7A0.3MnO3/PMN-PT(001) $(\mathrm{A}=\mathrm{Sr}, \mathrm{Ca})$. Phys. Rev. B 75, 054408 (2007).

14 Chakhalian, J., Freeland, J. W., Habermeier, H. U., Cristiani, G., Khaliullin, G. van Veenendaal, M. \& Keimer, B. Orbital reconstruction and covalent bonding at an oxide interface. Science 318, 1114-1117 (2007).

15 Huijben, M., Martin, L. W., Chu, Y.-H., Holcomb, M. B., Yu, P., Rijnders, G., Blank, D. H.A. \& Ramesh, R. Critical thickness and orbital ordering in ultrathin La0.7SrO.3MnO3 films. Phys. Rev. B 78, 094413 (2008).

16 Lai, K., Nakamura, M., Kundhikanjana, W., Kawasaki, M., Tokura, Y., Kelly, M. A. \& Shen, Z. X. Mesoscopic percolating resistance network in a strained manganite thin film. Science 329, 190-193 (2010).

17 Kourkoutis, L. F., Song, J. H., Hwang, H. Y. \& Muller, D. A. Microscopic origins for stabilizing room-temperature ferromagnetism in ultrathin manganite layers. Proc. Nat/ Acad. Sci. 107, 11682-11685 (2010).

18 Pesquera, D., Herranz, G., Barla, A., Pellegrin, E., Bondino, F., Magnano, E., Sánchez, F. \& Fontcuberta, J. Surface symmetry-breaking and strain effects on orbital occupancy in transition metal perovskite epitaxial films. Nat. Commun. 3, 1189 (2012).

19 Boschker, H., Verbeeck, Jo, Egoavil, Ricardo, Bals, Sara, Tendeloo, Gustaf van, Huijben, Mark, Houwman, Evert P., Koster, Gertjan, Blank, Dave H.A. \& Rijnders, Guus Preventing the reconstruction of the polar discontinuity at oxide heterointerfaces. Adv. Funct. Mater. 22, 2235-2240 (2012).

20 Wang, B., You, L., Ren, P., Yin, X., Peng, Y., Xia, B., Wang, L., Yu, X., Poh, S. M. Yang, P., Yuan, G., Chen, L., Rusydi, A. \& Wang, J. Oxygen-driven anisotropic transport in ultra-thin manganite films. Nat. Commun. 4, 2778 (2013).

21 Zhai, X., Cheng, L., Liu, Y., Schlepütz, C. M., Dong, S., Li, H., Zhang, X., Chu, S., Zheng, L., Zhang, J., Zhao, A., Hong, H., Bhattacharya, A., Eckstein, J. N. \& Zeng, C. Correlating interfacial octahedral rotations with magnetism in $(\mathrm{LaMnO} 3+\delta) \mathrm{N} /(\mathrm{SrTiO}) \mathrm{N}$ superlattices. Nat. Commun. 5, 4283 (2014).

22 Asmara, T. C., Annadi, A., Santoso, I., Gogoi, P. K., Kotlov, A., Omer, H. M., Motapothula, M., Breese, M. B., Rübhausen, M., Venkatesan, T., Ariando \& Rusydi, A. Mechanisms of charge transfer and redistribution in LaAIO3/SrTiO3 revealed by high-energy optical conductivity. Nat. Commun. 5, 3663 (2014).

23 Rusydi, A., Rauer, R., Neuber, G., Bastjan, M., Mahns, I., Müller, S., Saichu, P., Schulz, B., Singer, S. G., Lichtenstein, A. I., Qi, D., Gao, X., Yu, X., Wee, A. T.S., Strayganyuk, G., Dörr, K., Sawatzky, G. A., Cooper, S. L. \& Rübhausen, M. Metal-insulator transition in manganites: changes in optical conductivity up to $22 \mathrm{eV}$. Phys. Rev. B 78, 125110 (2008).

24 Tebano, A., Aruta, C., Sanna, S., Medaglia, P. G., Balestrino, G., Sidorenko, A. A., De Renzi, R., Ghiringhelli, G., Braicovich, G., Bisogni, V. \& Brookes, N. B. Evidence of orbital reconstruction at interfaces in ultrathin La0.67SrO.33MnO3 films. Phys. Rev. Lett. 100, 137401 (2008).

25 Stöhr, J. \& König, H. Determination of spin- and orbital-moment anisotropies in transition metals by angle-dependent X-ray magnetic circular dichroism. Phys. Rev. Lett. 75, 3748-3751 (1995).

26 Koide, T., Miyauchi, H., Okamoto, J., Shidara, T., Fujimori, A., Amemiya, K., Takeshita, H., Yuasa, S., Katayama, T. \& Suzuki, Y. Direct determination of interfacial magnetic moments with a magnetic phase transition in Co nanoclusters on $\mathrm{Au}(111)$. Phys. Rev. Lett. 87, 257201 (2001)

27 Arima, T., Tokura, Y. \& Torrance, J. B. Variation of optical gaps in perovskite-type 3d transition-metal oxides. Phys. Rev. B 48, 17006-17009 (1993).

28 Okimoto, Y., Katsufuji, T., Ishikawa, T., Arima, T. \& Tokura, Y. Variation of electronic structure in La1-xSrxMnO3 $(0 \leq x \leq 0.3)$ as investigated by optical conductivity spectra. Phys. Rev. B 55, 4206-4214 (1997).
29 Rauer, R., Rübhausen, M. \& Dörr, K. Magnetic-order induced spectral-weight redistribution in La0.7(Sr, Ca)0.3Mn03. Phys. Rev. B 73, 092402 (2006).

30 Moritomo, Y., Machida, A., Matsuda, K., Ichida, M. \& Nakamura, A. Magnetizationdependent behaviors of interband transitions between the exchange-split bands in doped manganite films. Phys. Rev. B 56, 5088-5091 (1997).

31 Quijada, M., Černe, J., Simpson, J. R., Drew, H. D., Ahn, K. H., Millis, A. J., Shreekala, R., Ramesh, R., Rajeswari, M. \& Venkatesan, T. Optical conductivity of manganites: crossover from Jahn-Teller small polaron to coherent transport in the ferromagnetic state. Phys. Rev. B 58, 16093-16102 (1998).

32 Takenaka, K., Sawaki, Y., Shiozaki, R. \& Sugai, S. Electronic structure of the doubleexchange ferromagnet La0.825Sr0.175MnO3 studied by optical reflectivity. Phys. Rev. B 62, 13864-13867 (2000).

33 Meinders, M. B.J., Eskes, H. \& Sawatzky, G. A. Spectral-weight transfer: breakdown of low-energy-scale sum rules in correlated systems. Phys. Rev. B 48, 3916-3926 (1993).

34 Majidi, M. A., Su, H., Feng, Y. P., Rübhausen, M. \& Rusydi, A. Theory of high-energy optical conductivity and the role of oxygens in manganites. Phys. Rev. B 84, 075136 (2011).

35 Abbate, M., de Groot, F. M., Fuggle, J. C., Fujimori, A., Strebel, O., Lopez, F., Domke, M., Kaindl, G., Sawatzky, G. A., Takano, M., Takeda, Y., Eisaki, H. \& Uchida, S. Controlled-valence properties of La1-xSrxFeO3 and La1-xSrxMnO3 studied by soft-x-ray absorption spectroscopy. Phys. Rev. B 46, 4511-4519 (1992).

36 Thole, B. T., Carra, P., Sette, F. \& van der Laan, G. X-ray circular dichroism as a probe of orbital magnetization. Phys. Rev. Lett. 68, 1943-1946 (1992).

37 Carra, P., Thole, B. T., Altarelli, M. \& Wang, X. X-ray circular dichroism and local magnetic fields. Phys. Rev. Lett. 70, 694-697 (1993).

38 Pellegrin, E., Tjeng, L. H., de Groot, F. M.F., Hesper, R., Sawatzky, G. A., Moritomo, Y. \& Tokura, Y. Soft X-ray magnetic circular dichroism study of the colossal magnetoresistance compound La1-xSrxMnO3. J. Electron. Spectrosc. Relat. Phenom. 86, 115-118 (1997)

39 Konishi, Y., Fang, Z., Izumil, M., Manako, T., Kasail, M., Kuwahara, H., Kawasaki, M., Terakura, K. \& Tokura, Y. Orbital-state-mediated phase-control of manganites. J. Phys. Soc. Jpn. 68, 3790-3793 (1999).

40 Koide, T., Miyauchi, H., Okamoto, J., Shidara, T., Sekine, T., Saitoh, T., Fujimori, A., Fukutani, H., Takano, M. \& Takeda, Y. Close correlation between the magnetic moments, lattice distortions, and hybridization in $\mathrm{LaMnO3}$ and La1-xSrxMnO3+delta: doping-dependent magnetic circular X-ray dichroism study. Phys. Rev. Lett. 87, 246404 (2001).

41 de Gennes, P. G. Effects of double exchange in magnetic crystals. Phys Rev 118, 141-154 (1960)

42 Dagotto, E., Hotta, T. \& Moreo, A. Colossal magnetoresistant materials: the key role of phase separation. Phys. Rep. 344, 1-153 (2001).

43 Burkhardt, M. H., Hossain, M. A., Sarkar, S., Chuang, Y. D., Cruz Gonzalez, A. G., Doran, A., Scholl, A., Young, A. T., Tahir, N., Choi, Y. J., Cheong, S. W., Dürr, H. A. \& Stöhr, J. Imaging the first-order magnetic transition in La0.35Pr0.275Ca0.375MnO3. Phys. Rev. Lett. 108, 237202 (2012).

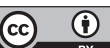

This work is licensed under a Creative Commons Attribution 4.0 International License. The images or other third party material in this article are included in the article's Creative Commons license, unless indicated otherwise in the credit line; if the material is not included under the Creative Commons license, users will need to obtain permission from the license holder to reproduce the material. To view a copy of this license, visit http:// creativecommons.org/licenses/by/4.0/

Supplementary Information accompanies the paper on the NPG Asia Materials website (http://www.nature.com/am) 